\title{
Color Current Induced by Gluon in Background Field Method of QCD
}

\author{
Qun Wang ${ }^{1,2}$, Chung-Wen Kao ${ }^{1}$, Gouranga C. Nayak ${ }^{1}$, Horst Stöcker ${ }^{1}$ and Walter Greiner ${ }^{1}$ \\ ${ }^{1}$ Institut für Theoretische Physik, J. W. Goethe-Universität, 60054 Frankfurt am Main, Germany \\ ${ }^{2}$ Physics Department, Shandong University, Jinan, Shandong 250100, P. R. China
}

(October 31, 2018)

\begin{abstract}
By using the background field method of QCD in a path integral approach, we derive the equation of motion for the classical chromofield and for the gluon in a system containing the gluon and the classical chromofield simultaneously. This inhomogeneous field equation contains a current term, which is the expectation value of a composite operator including linear, square and cubic terms of the gluon field. We also derive identities which the current should obey from the gauge invariance. We calculate the current at the leading order where the current induced by the gluon is opposite in sign to that induced by the quark. This is just the feature of the non-Abelian gauge field theory which has asymptotic freedom. Physically, the induced current can be treated as the 'displacement' current in the polarized vacuum, and its effect is equivalent to redefining the field and the coupling constant.
\end{abstract}

PACS: 12.38.-t,12.38.Aw,11.15.-q,12.38.Mh

\section{INTRODUCTION}

The behavior of electric charged particles in a classical electromagnetic field has been extensively studied in the literature. However, the behavior of color charged particles (quarks and gluons) in a classical chromofield has been less studied. The gluon case is particularly interesting because there is no analogue in the electromagnetic theory, since photons do not interact with the classical electromagnetic field. One important issue is to derive the equation of motion for the gluon in the presence of chromofield and to define and calculate the classical current produced by the gluon. As we know it is easy to define and understand the color current for quarks in the classical chromofield. But strictly defining the color current for gluons classically is not an easy task, mainly due to its quantum and bosonic nature. We plan to address some aspects of these issues in this paper.

At first sight, this problem looks as if it were only of theoretical interest. However, it turns out to be applicable to a non-equilibrium system of quarks and gluons, which is expected to be produced in ultra-relativistic heavy ion collisions in RHIC and LHC where a classical chromofield may be produced. The dynamics of a quantum non-equilibrium manybody system is usually studied in the closed-time-path formulation with non-local source 
terms 回. This formalism needs to be extended by incorporating classical chromofield. The evolution of partons can be obtained by solving the Schwinger-Dyson equation defined on a closed-time-path. This is formidable task because it involves a non-local non-linear integrodifferential equation and because of its quantum nature. There are two scales in the system: the quantum (microscopic) scale and the statistical-kinetic (macroscopic) one. When the statistical-kinetic scale is much larger than the quantum one, the Schwinger-Dyson equation may be recasted into a much simpler form of the kinetic Boltzmann equation by a gradient expansion. The Boltzmann equation describes evolution of the particle distribution function in the phase space of momentum-coordinate, and can be solved numerically for practical purpose. For a non-equilibrium system of quarks and gluons in a chromofield, the distribution function also depends on the classical color charge of the parton, since the color is exchanged between the chromofield and partons and among partons themselves. In this case, the Boltzmann equation also describes the evolution of the parton distribution function in the color space [2, [2]. While the Boltzmann equation describes the kinetic and color evolution of the hard parton system, soft partons are normally treated as a coherent classical field whose evolution may be described by a equation which is similar to YangMills equation. Therefore, one should study the transport problem for hard partons in the presence of a classical background field. For example, in high energy heavy-ion collisions, minijets (which are hard partons) are initially produced and then propagate in a classical chromofield created by the soft partons [4 [6]. In this situation it is necessary to derive the equation of the gluon and the classical chromofield to study the formation and equilibration of quark-gluon plasma. Hence we see that the issues we want to address in this paper have practical implication in ultra-relativistic heavy ion collisions.

Since conventional QCD does not contain classical chromofield, to include the classical chromofield in a proper way, we follow the background field method of QCD (BG-QCD). This method was first introduced by DeWitt and 't Hooft [7,8]. The advantage of BG-QCD is that it is formulated in an explicit gauge invariance manner(see below). This method is widely used to study confinement, gravity and hot quark-gluon-plasma, etc.. In this paper, we derive the equation of motion for the gluon and the classical background field to define the induced current in a path integral approach which fully incorporate non-Abelian gauge symmetry. Also some properties of the induced current are given in the framework of BG-QCD.

The paper is organized as follows. In section II, we briefly introduce basics of BG-QCD and the Ward identity which we will use later. In section III, equations of motion for the classical and the gluon field are derived, and the current induced by the gluon is defined. We study its property and derive the Ward identity that the current obeys in the next section. Section V presents the result for the current at the leading order. The physical implication for the result is also discussed. The summary and conclusion is presented in the last section.

\section{BACKGROUND FIELD METHOD}

We know that any physical quantity calculated in the conventional QCD is gauge invariant and independent of the particular gauge chosen. However, there is still a problem of gauge invariance which is evident in the off shell Green's functions and counter terms. This

problem arises because one must fix a gauge and introduce the gauge-fixing and ghost term 
to quantize the theory. The classical Lagrangian for the gauge field has explicit gauge is invariant while the gauge-fixing and ghost term is not gauge invariant although it is invariant under the BRS transformation. The background field method is such a technique which allows one to fix a gauge (background-field gauge) without losing explicit gauge invariance which is present in the classical level of the gauge field theory.

By writing the conventional gluon field into the sum of a classical background part and the quantum one, the action in the background field gauge is given by [9] 113]:

$$
\begin{aligned}
& S=S_{0}+S_{f i x}+S_{\text {ghost }}+S_{\text {source }} \\
& S_{0}=-\frac{1}{4} \int d^{4} x\left(F_{\mu \nu}^{i}[A]+D_{\mu}^{i j}[A] Q_{\nu}^{j}-D_{\nu}^{i j}[A] Q_{\mu}^{j}+g f^{i j k} Q_{\mu}^{j} Q_{\nu}^{k}\right)^{2}, \\
& S_{f i x}=-\frac{1}{2 \alpha} \int d^{4} x\left(D_{\mu}^{i j}[A] Q^{\mu, j}\right)^{2}, \\
& S_{\text {ghost }}=\int d^{4} x \bar{C}^{i} D_{\mu}^{i j}[A] D^{\mu, j k}[A+Q] C^{k}, \\
& S_{\text {source }}=\int d^{4} x\left(J_{\mu}^{i} Q^{\mu, i}+\bar{\xi}^{i} C^{i}+\bar{C}^{i} \xi^{i}\right) .
\end{aligned}
$$

The generating functional for Green's function is

$$
Z[A, J, \xi, \bar{\xi}]=\int[d Q][d C][d \bar{C}] \exp (i S)
$$

The variables are defined as follows: $A_{\mu}^{i}$ is the classical background field; $Q_{\mu}^{i}$ is the gluon field, the variable of integration in the functional integral; $C^{i}, \bar{C}^{i}$ are ghost and anti-ghost fields; $\bar{\xi}^{i}, \xi^{i}$ are external sources coupling to ghost and anti-ghost respectively. $D_{\mu}^{i j}[A]$ is the covariant derivative in the adjoint representation whose generators are $\left(t_{A}^{a}\right)^{i j}=i f^{i a j}$ and it is defined as $D_{\mu}^{i j}[A] \equiv \partial_{\mu} \delta^{i j}+g f^{i a j} A_{\mu}^{a}=\partial_{\mu} \delta^{i j}-i g\left(t_{A}^{a}\right)^{i j} A_{\mu}^{a} ; F_{\mu \nu}^{i}[A]$ is the field strength tensor for the background field and given by $F_{\mu \nu}^{i}[A]=\partial_{\mu} A_{\nu}^{i}-\partial_{\nu} A_{\mu}^{i}+g f^{i j k} A_{\mu}^{j} A_{\nu}^{k}$.

The gauge transformation for all fields and sources which leaves $Z$ invariant is given by:

$$
\begin{aligned}
& A_{\mu}^{\prime}=U A_{\mu} U^{-1}+\frac{i}{g} U \partial_{\mu} U^{-1}, \\
& Q_{\mu}^{\prime}=U Q_{\mu} U^{-1}, \quad J_{\mu}^{\prime}=U J_{\mu} U^{-1}, \\
& C_{\mu}^{\prime}=U C U^{-1}, \quad \bar{\xi}^{\prime}=U \bar{\xi} U^{-1}, \\
& \bar{C}^{\prime}=U \bar{C} U^{-1}, \quad \xi^{\prime}=U \xi U^{-1} .
\end{aligned}
$$

This gauge transformation is known as Type-I transformation [11]. Note that the background field transforms in the conventional way while the gluon field, the ghost field and all external sources transform like a matter field. The infinitesimal changes for these fields and sources are:

$$
\begin{aligned}
& \delta A_{\mu}^{i}=D_{\mu}^{i j}[A] \omega^{j}, \\
& \delta Q_{\mu}^{i}=g f^{i j k} Q_{\mu}^{j} \omega^{k}, \quad \delta J_{\mu}^{i}=g f^{i j k} J_{\mu}^{j} \omega^{k}, \\
& \delta C^{i}=g f^{i j k} C^{j} \omega^{k}, \quad \delta \bar{\xi}^{i}=g f^{i j k} \bar{\xi}^{j} \omega^{k}, \\
& \delta \bar{C}^{i}=g f^{i j k} \bar{C}^{j} \omega^{k}, \quad \delta \xi^{i}=g f^{i j k} \xi^{j} \omega^{k} .
\end{aligned}
$$

Here we define the gauge transformation as $U(x)=\exp \left(i g \omega^{a}(x) t_{A}^{a}\right)$. Roman alphabets denote color indices and Greek ones denote the Lorentz indices. All fields and sources are either in the vector form, e.g. $A_{\mu} \equiv A_{\mu}^{a} t_{A}^{a}$, or with their color indices explicitly shown, e.g. $A_{\mu}^{a}$. It is obvious that the generating functional $Z$ is invariant under the above transformation. 
But the less obvious is the invariance of $S_{f i x}$. To prove it, one should remember the fact that $t_{A}^{a}$ is a pure imaginary Hermitian matrix and that $\left(t_{A}^{a}\right)^{T}=-t_{A}^{a}$, therefore $U^{T}=U^{-1}$.

The generating functional for the connected Green function is given by:

$$
W[A, J, \xi, \bar{\xi}]=-i \ln Z[A, J, \xi, \bar{\xi}]
$$

Field averages can be obtained by taking derivative of $W$ with respect to their corresponding sources:

$$
\left\langle Q_{\mu}^{i}\right\rangle=\frac{\delta W}{\delta J^{\mu, i}}, \quad\left\langle C^{i}\right\rangle=\frac{\delta W}{\delta \bar{\xi}^{i}}, \quad\left\langle\bar{C}^{i}\right\rangle=-\frac{\delta W}{\delta \xi^{i}}
$$

The one-particle irreducible generating functional $\Gamma[A,\langle Q\rangle,\langle C\rangle,\langle\bar{C}\rangle]$ is defined by Legendre transformation for $W$ :

$$
\Gamma[A,\langle Q\rangle,\langle C\rangle,\langle\bar{C}\rangle]=W[A, J, \xi, \bar{\xi}]-\int d^{4} x\left(J_{\mu}^{i}\left\langle Q^{\mu, i}\right\rangle+\bar{\xi}^{i}\left\langle C^{i}\right\rangle+\left\langle\bar{C}^{i}\right\rangle \xi^{i}\right)
$$

where we see that $\frac{\delta \Gamma}{\delta A_{\mu}^{j}}=\frac{\delta W}{\delta A_{\mu}^{j}}$. Since $\Gamma[A,\langle Q\rangle,\langle C\rangle,\langle\bar{C}\rangle]$ is a gauge invariant functional of $A$, $\langle Q\rangle,\langle C\rangle$, and $\langle\bar{C}\rangle$, hence $\Gamma[A,\langle Q\rangle,\langle C\rangle,\langle\bar{C}\rangle]$ should be invariant under the transformation (田). Then we have the following Ward identity [11:

$$
D_{\mu}^{i j}[A] \frac{\delta \Gamma}{\delta A_{\mu}^{j}}+g f^{i j k}\left(\left\langle Q_{\mu}^{j}\right\rangle \frac{\delta \Gamma}{\delta\left\langle Q_{\mu}^{k}\right\rangle}+\left\langle C^{j}\right\rangle \frac{\delta \Gamma}{\delta\left\langle C^{k}\right\rangle}+\left\langle\bar{C}^{j}\right\rangle \frac{\delta \Gamma}{\delta\left\langle\bar{C}^{k}\right\rangle}\right)=0
$$

In the same way, we have the parallel Ward identity in terms of $W$ :

$$
D_{\mu}^{i j}[A] \frac{\delta W}{\delta A_{\mu}^{j}}+g f^{i j k}\left(J_{\mu}^{j} \frac{\delta W}{\delta J_{\mu}^{k}}+\xi^{j} \frac{\delta W}{\delta \xi^{k}}+\bar{\xi}^{j} \frac{\delta W}{\delta \bar{\xi}^{k}}\right)=0 .
$$

Note that Eq.(8) and (9) are identical because $\frac{\delta \Gamma}{\delta A_{\mu}^{j}}=\frac{\delta W}{\delta A_{\mu}^{j}}$.

\section{EQUATION OF MOTION FOR GLUON AND CLASSICAL FIELD AND DEFINITION OF INDUCED CURRENT}

By shifting the quantum field $Q_{\mu}^{i}(x)$, a variable of integration in the functional integral, by a small amount $v_{\mu}^{i}(x): Q_{\mu}^{i}(x)=Q_{\mu}^{i}(x)+v_{\mu}^{i}(x)$, we can obtain the equation of motion or the Lagrange equation for the quantum field $Q_{\mu}^{i}$. The change of the functional for connected Green's functions $W$ is:

$$
\delta W=-i \delta \ln Z=Z^{-1} \int[d Q][d C][d \bar{C}] \int d^{4} x \frac{\delta S}{\delta Q_{\mu}^{i}} v_{\mu}^{i}(x) \exp (i S) .
$$

The fact that $W$ should be independent of the shifting value of the integration variable leads to the equation of motion for $Q_{\mu}^{i}$ :

$$
\left\langle\frac{\delta S}{\delta Q_{\mu}^{i}(x)}\right\rangle=Z^{-1} \int[d Q][d C][d \bar{C}]\left(\frac{\delta\left(S_{0}+S_{\text {fix }}+S_{\text {ghost }}\right)}{\delta Q_{\mu}^{i}(x)}+J^{\mu, i}(x)\right) \exp (i S)=0 .
$$


In the absence of the external source $J_{\mu}^{i}$, the equation of motion becomes:

$$
\left\langle\frac{\delta S_{0}}{\delta Q_{\mu}^{i}}\right\rangle+\left\langle\frac{\delta S_{f i x}}{\delta Q_{\mu}^{i}}\right\rangle+\left\langle\frac{\delta S_{\text {ghost }}}{\delta Q_{\mu}^{i}}\right\rangle=0,
$$

where we have

$$
\begin{aligned}
& \frac{\delta S_{0}}{\delta Q_{\mu}^{i}}=D_{\nu}^{i j}[A+Q] F^{\nu \mu, j}[A+Q] \\
& \frac{\delta S_{f i x}}{\delta Q_{\mu}^{i}}=\frac{1}{\alpha} D^{\mu, i j}[A] D^{\nu, j k}[A] Q_{\nu}^{k} \\
& \frac{\delta S_{\text {ghost }}}{\delta Q_{\mu}^{i}}=-g f^{k i l} \bar{C}^{j} \overleftarrow{D}^{\mu, k j}[A] C^{l}
\end{aligned}
$$

Note that the partial derivative operation in $\overleftarrow{D}^{\mu, k j}$ is toward $\bar{C}^{j}$. We know that $F^{\nu \mu, j}[A+Q]$ transforms like a matter field and $D_{\nu}^{i j}[A+Q]$ is commutable with the transformation matrix. So the first equation $\frac{\delta S_{0}}{\delta Q_{\mu}^{i}}=D_{\nu}^{i j}[A+Q] F^{\nu \mu, j}[A+Q]$ transforms like a matter field in adjoint representation. Similarly we can easily verify that the second equation transforms like a matter field. But it is less apparent to verify that the ghost part $\frac{\delta S_{\text {ghost }}}{\delta Q_{\mu}^{i}}$ also transforms like a matter field. Let us verify it directly. The variation of $\frac{\delta S_{\text {ghost }}}{\delta Q_{\mu}^{i}}$ under the infinitesimal gauge transformation Eq.(四) is:

$$
\begin{aligned}
& \delta\left(\frac{\delta S_{\text {ghost }}}{\delta Q_{\mu}^{i}}\right)=-g f^{k i l} \delta\left(\bar{C}^{j} \overleftarrow{D}^{\mu, k j}[A]\right) C^{l}-g f^{k i l} \bar{C}^{j} \overleftarrow{D}^{\mu, k j}[A] \delta C^{l} \\
& =-g f^{a i d}\left(g f^{a k e} \bar{C}^{j} \overleftarrow{D}^{\mu, k j}[A] \omega^{e}\right) C^{d}-g f^{k i l} \bar{C}^{j} \overleftarrow{D}^{\mu, k j}[A]\left(g f^{l d e} C^{d} \omega^{e}\right) \\
& =-g^{2}\left(f^{a i d} f^{a k e}+f^{k i l} f^{l d e}\right) \bar{C}^{j} \overleftarrow{D}^{\mu, k j}[A] C^{d} \omega^{e} \\
& =g f^{i l e}\left[-g f^{k l d} \bar{C}^{j} \overleftarrow{D}^{\mu, k j}[A] C^{d}\right] \omega^{e}
\end{aligned}
$$

Therefore we see that $\frac{\delta S_{\text {ghost }}}{\delta Q_{\mu}^{i}}$ transforms like a matter field in adjoint representation.

We can rewrite Eq.(13) in another form:

$$
D_{\nu}^{i j}[A] F^{\nu \mu, j}[A]=\left\langle j_{0}^{\mu, i}\right\rangle
$$

where the current $j_{0}$ induced by the quantum field is given by:

$$
\begin{aligned}
j_{0}^{\mu, i}= & -g f^{a b c} D_{\nu}^{i a}[A]\left(Q^{\nu, b} Q^{\mu, c}\right)-g f^{i d a} Q_{\nu}^{d}\left(D^{\nu, a b}[A] Q^{\mu, b}-D^{\mu, a b}[A] Q^{\nu, b}\right) \\
& -g^{2} f^{i d a} f^{a b c} Q_{\nu}^{d} Q^{\nu, b} Q^{\mu, c}-D_{\nu}^{i j}[A]\left(D^{\nu, j k}[A] Q^{\mu, k}-D^{\mu, j k}[A] Q^{\nu, k}\right)-g f^{i j k} Q_{\nu}^{j} F^{\nu \mu, k}[A] \\
& +g f^{k i l} \bar{C}^{j} \overleftarrow{D}^{\mu, k j}[A] C^{l}-\frac{1}{\alpha} D^{\mu, i j}[A] D^{\nu, j k}[A] Q_{\nu}^{k}
\end{aligned}
$$

The last two terms in $j_{0}$ are just $-\delta\left(S_{\text {fix }}+S_{\text {ghost }}\right) / \delta Q$. Obviously $j_{0}$ transforms like a matter field.

It is easy to check $\delta S_{0} / \delta Q=\delta S_{0} / \delta A$. But it takes some steps to prove:

$$
\left\langle\frac{\delta\left(S_{f i x}+S_{\text {ghost }}\right)}{\delta Q_{\mu}^{i}}\right\rangle=\left\langle\frac{\delta\left(S_{f i x}+S_{\text {ghost }}\right)}{\delta A_{\mu}^{i}}\right\rangle .
$$

We start with $Z[A, 0,0,0]$ where in Eq.(2) the sources are set to zero. We shift $A$ by $\delta A$ and $Q$ by $-\delta A$ so that $A+Q$ does not change. $S_{0}$ is invariant in this operation. The variation of $Z[A, 0,0,0]$ is caused by that of $\left(S_{\text {fix }}+S_{\text {ghost }}\right)$ : 


$$
\begin{aligned}
\delta Z[A, 0,0,0]= & i \int[d Q][d C][d \bar{C}]\left\{\frac{\delta\left(S_{\text {fix }}+S_{\text {ghost }}\right)}{\delta A_{\mu}^{i}}-\frac{\delta\left(S_{\text {fix }}+S_{\text {ghost }}\right)}{\delta Q_{\mu}^{i}}\right\} \delta A_{\mu}^{i} \exp (i S) \\
= & i \int[d Q][d C][d \bar{C}]\left\{-\frac{1}{\alpha} D^{\mu, i j}[A+Q] D^{\nu, j k}[A] Q_{\nu}^{k}\right. \\
& \left.-g f^{i j k} \bar{C}^{j} D^{\mu, k l}[A+Q] C^{l}\right\} \delta A_{\mu}^{i} \exp (i S)
\end{aligned}
$$

where $S=S_{0}+S_{f i x}+S_{\text {ghost }}$. We expect that it would leave $Z[A, 0,0,0]$ unchanged i.e. $\delta Z[A, 0,0,0] / \delta A_{\mu}^{i}=0$, since nothing happens for $S_{0}[A+Q]$. But it is non-trivial to prove this. We provide here a proof based on Ref. [11] and [12]. Consider a kind of special infinitesimal gauge transformation defined by: $\delta Q_{\mu}^{i}=D_{\mu}^{i j}[A+Q] \omega^{j}$ where the infinitesimal gauge group parameter $\omega^{j}(x)$ is defined by:

$$
\omega^{j}(x)=M^{-1, j k}(x, y) D_{\nu}^{k l}[A+Q](y) \delta A_{\nu}^{l}(y)
$$

where the repetition of indices implies the summation or the integration; The matrix $M$ is just the one which couples to the ghost field:

$$
M^{i j}(x, y)=D_{\mu}^{i k}[A] D_{\nu}^{k j}[A+Q](x) \delta(x-y)
$$

we have following properties:

$$
D_{\mu}^{i j}[A](x) \delta Q_{\mu}^{j}(x)=M^{i k}(x, y) \omega^{k}(y)=D_{\mu}^{i j}[A+Q](x) \delta A_{\mu}^{j}(x)
$$

We say this infinitesimal gauge transformation is special because it can make gauge condition invariant in some circumstances. In this paper, we imply the covariant gauge constraint $\delta\left(D_{\mu}^{i j}[A] Q^{\mu, j}-a^{i}\right)$ in the generating functional $Z$. If we increase $a^{i}$ by a small amount $\delta a^{i}$ and simultaneously make the above gauge transformation where the small parameter $\omega^{k}(y)$ is given the value so that $M^{i k}(x, y) \omega^{k}(y)=\delta a^{i}$, the gauge constraint remains the same. In fact the new gauge constraint

$$
\begin{aligned}
\delta\left(D_{\mu}^{i j}[A] Q^{\prime \mu, j}-a^{i i}\right) & =\delta\left(D_{\mu}^{i j}[A] Q^{\mu, j}+D_{\mu}^{i j}[A] \delta Q_{\mu}^{j}-a^{i}-\delta a^{i}\right) \\
& =\delta\left(D_{\mu}^{i j}[A] Q^{\mu, j}-a^{i}\right)
\end{aligned}
$$

is unchanged. The other part of the generating functional $Z$ can be proved to be invariant under the above gauge transformation constrained by $M^{i k}(x, y) \omega^{k}(y)=\delta a^{i}$. Hence $Z$ is independent of $a^{i}$. One can therefore multiplies an arbitrary function of $a^{i}$ and integrate over $a^{i}$ without changing the result up to some irrelevant normalization constant. Usually this function of $a^{i}$ is chosen to be a Gaussian function $\exp \left(-i \int d^{4} x\left(a^{i}(x)\right)^{2} / 2 \alpha\right)$ which gives the gauge fixing term $S_{f i x}$. One can prove that the variation $\delta Z$ caused by the above defined gauge transformation constrained by $M^{i k}(x, y) \omega^{k}(y)=\delta a^{i}$ is just $\delta Z[A, 0,0,0]$ in Eq.(18) which is zero. Therefore we obtain $\left\langle\delta\left(S_{\text {fix }}+S_{\text {ghost }}\right) / \delta A\right\rangle=\left\langle\delta\left(S_{\text {fix }}+S_{\text {ghost }}\right) / \delta Q\right\rangle$. Together with $\delta S_{0} / \delta Q=\delta S_{0} / \delta A$, we have

$$
\left\langle\delta\left(S_{0}+S_{\text {fix }}+S_{\text {ghost }}\right) / \delta A\right\rangle=\left\langle\delta\left(S_{0}+S_{\text {fix }}+S_{\text {ghost }}\right) / \delta Q\right\rangle=0
$$

From $\left\langle\delta\left(S_{0}+S_{\text {fix }}+S_{\text {ghost }}\right) / \delta A\right\rangle=0$, we obtain:

$$
D_{\nu}^{i j}[A] F^{\nu \mu, j}[A]=\left\langle j^{\mu, i}\right\rangle,
$$

where $j$ is defined as: 


$$
\begin{aligned}
j^{\mu, i}= & -g f^{a b c} D_{\nu}^{i a}[A]\left(Q^{\nu, b} Q^{\mu, c}\right)-g f^{i d a} Q_{\nu}^{d}\left(D^{\nu, a b}[A] Q^{\mu, b}-D^{\mu, a b}[A] Q^{\nu, b}\right) \\
& -g^{2} f^{i d a} f^{a b c} Q_{\nu}^{d} Q^{\nu, b} Q^{\mu, c}-D_{\nu}^{i j}[A]\left(D^{\nu, j k}[A] Q^{\mu, k}-D^{\mu, j k}[A] Q^{\nu, k}\right)-g f^{i j k} Q_{\nu}^{j} F^{\nu \mu, k}[A] \\
& -g f^{k i l} \bar{C}^{j} \stackrel{\leftrightarrow}{D}^{\mu, k j}[A] C^{l}-g f^{i j k} \bar{C}^{j} D^{\mu, k l}[A+Q] C^{l}+\frac{1}{\alpha} g f^{i j k} Q^{\mu, k} D_{\nu}^{j l}[A] Q^{\nu, l}
\end{aligned}
$$

where the last three terms are just $\delta\left(S_{f i x}+S_{\text {ghost }}\right) / \delta A_{\mu}^{i}$. Because of the identity $D_{\mu}^{i j}[A] D_{\nu}^{j k}[A] F^{\nu \mu, k}[A]=0$, we have $D_{\mu}^{i j}[A]\left\langle j^{\mu, j}\right\rangle=0$.

Eq.(15), Eq.(17) and Eq.(24) are our main results. They guarantee the consistent definition of the induced current. As we see it is easier and clearer to prove the equation of motion for $\langle Q\rangle,\left\langle\frac{\delta S}{\delta Q}\right\rangle=0$. But it is less apparent for $\left\langle\frac{\delta S}{\delta A}\right\rangle=0$, the equation of motion for the classical background field, in a quantum field formalism. As a contrast, it is straightforward to obtain the Lagrange equation of motion for a field in the classical field theory, but the current situation is subtle because the treatment of the quantum field, the gauge fixing term as well as the ghost field and is highly non-trivial. The proof for the equivalence of two equations is guaranteed by the intrinsic relation between the the gauge fixing term and the ghost sector, or in other words, the equivalence and the consistency of two equations is a natural outcome of the symmetry behind the non-Abelian gauge field theory. We notice that Ref. [13] also derives Eq.(24) from a canonical formalism, but what we use here is a path integral approach where the symmetry of non-Abelian gauge field is explicitly fulfilled and exploited.

\section{IDENTITY FOR INDUCED CURRENT}

We now try to derive the equation for the current from the Ward identity. In order to derive the equation for the current from the Ward identity, we add to $S_{\text {source }}$ a new source term $\int d^{4} x Y_{\mu}^{i} j^{\mu, i}$ where the current $j$ induced by the quantum field couples to its external source $Y$. Since $j$ transforms like a matter field, we require that $Y$ also transform in the same way to ensure the gauge invariance of the new source term.

Before we proceed, some points in the derivation should be clarified. We distinguish two cases:

Case I. There is no constraint on values of $\left\langle Q_{\mu}^{i}\right\rangle=\delta W / \delta J^{\mu, i},\left\langle C^{i}\right\rangle=\delta W / \delta \overline{\xi^{i}}$ and $\left\langle\bar{C}^{i}\right\rangle=-\delta W / \delta \xi^{i}$ in intermediate steps. Therefore, during the derivation there is no relation between external sources $(J, \xi, \bar{\xi})$ and the background field $A$. Hence sources and the background field are independent variables. Because we do not impose any specific value on $\langle j\rangle$ in the whole process, the source $Y$ remains irrelevant of $A$.

Case II. $\langle Q\rangle,\langle C\rangle$ and $\langle\bar{C}\rangle$ are set to zero in each step, as assumed by many other authors [9, [10], hence specific relations between sources $(J, \xi, \bar{\xi})$ and $A$ are built up by $\delta W[J, \xi, \bar{\xi}] / \delta J^{\mu, i}=0, \delta W / \delta \overline{\xi^{i}}=0$ and $\delta W / \delta \xi^{i}=0$. Each source is a functional of $A$ and vice versa. Through $A$, there are functional mappings between any two sources too. These sources are no longer independent of each other. But we do not assume any specific value for $\langle j\rangle$, so the source $Y$ is still independent of $A$. In this case, we have two independent variables, $Y$ and $A$.

So in both cases $Y$ is always an independent variable. We will see in either case, i.e. whether $\langle Q\rangle,\langle C\rangle,\langle\bar{C}\rangle=0$ or not, the equation for $\langle j\rangle$ is the same, though the Ward iden- 
tity for each case is different. Here we assume case I(For case II, see Appendix A). After introducing a new source term, the Ward identity (9) is extended to:

$$
D_{\mu}^{i j}[A] \frac{\delta W}{\delta A_{\mu}^{j}}+g f^{i j k}\left(Y_{\mu}^{j} \frac{\delta W}{\delta Y_{\mu}^{k}}+J_{\mu}^{j} \frac{\delta W}{\delta J_{\mu}^{k}}+\xi^{j} \frac{\delta W}{\delta \xi^{k}}+\bar{\xi}^{j} \frac{\delta W}{\delta \bar{\xi}^{k}}\right)=0
$$

where $\delta W / \delta A_{\mu}^{j}$ is given by:

$$
\begin{aligned}
\frac{\delta W}{\delta A_{\mu}^{j}} & =\left\langle\frac{\delta\left(S_{0}+S_{\text {fix }}+S_{\text {ghost }}\right)}{\delta A_{\mu}^{j}}\right\rangle+Y_{\nu}^{i}\left\langle\frac{\delta j^{\nu, i}}{\delta A_{\mu}^{j}}\right\rangle \\
& =D_{\nu}^{j k}[A] F^{\nu \mu, k}[A]-\left\langle j^{\mu, j}\right\rangle+Y_{\nu}^{k}\left\langle\frac{\delta j^{\nu, k}}{\delta A_{\mu}^{j}}\right\rangle .
\end{aligned}
$$

Substituting Eq.(27) into Eq.(26) and using the identity $D_{\mu}^{i j}[A] D_{\nu}^{j k}[A] F^{\nu \mu, k}[A]=0$, Eq.(26) becomes:

$$
-D_{\mu}^{i j}[A]\left\langle j^{\mu, j}\right\rangle+D_{\mu}^{i j}[A] Y_{\nu}^{k}\left\langle\frac{\delta j^{\nu, k}}{\delta A_{\mu}^{j}}\right\rangle+g f^{i j k}\left(Y_{\mu}^{j} \frac{\delta W}{\delta Y_{\mu}^{k}}+J_{\mu}^{j} \frac{\delta W}{\delta J_{\mu}^{k}}+\xi^{j} \frac{\delta W}{\delta \xi^{k}}+\bar{\xi}^{j} \frac{\delta W}{\delta \bar{\xi}^{k}}\right)=0 .
$$

Because we want to go further, we must keep all sources in Eq.(28). If we set all sources vanish at this stage, we get $D_{\mu}^{j k}[A]\left\langle j^{\mu, k}\right\rangle=0$ which we obtain in the former section. Taking derivative with respect to $Y_{\nu}^{k}(y)$ for Eq.(28), setting all sources vanish, and using $D_{\mu}^{j k}[A]\left\langle j^{\mu, k}\right\rangle=0$, we obtain:

$$
\begin{aligned}
& D_{\mu}^{i j}[A](x)\left\langle T\left[j^{\mu, j}(x) j^{\nu, k}(y)\right]\right\rangle+i \delta^{4}(x-y) g f^{i l j} A_{\mu}^{l}(x)\left\langle\frac{\delta j^{\nu, k}}{\delta A_{\mu}^{j}}(x)\right\rangle \\
& +i g \delta^{4}(x-y) f^{i k l}\left\langle j^{\nu, l}(x)\right\rangle+i \partial_{\mu}\left[\delta^{4}(x-y)\left\langle\frac{\delta j^{\nu, k}}{\delta A_{\mu}^{i}}(x)\right\rangle\right]=0 .
\end{aligned}
$$

Integrating Eq.(29) over $x$, and noting that the complete differential vanishes, we obtain:

$$
f^{i l j} \int d^{4} x A_{\mu}^{l}(x)\left\langle T\left[j^{\mu, j}(x) j^{\nu, k}(y)\right]\right\rangle+i f^{i l j} A_{\mu}^{l}(y)\left\langle\frac{\delta j^{\nu, k}}{\delta A_{\mu}^{j}}(y)\right\rangle+i f^{i k l}\left\langle j^{\nu, l}(y)\right\rangle=0 .
$$

In addition, integrating $D_{\mu}^{j k}[A]\left\langle j^{\mu, k}(x)\right\rangle=0$ over $x$, we obtain:

$$
\int d^{4} x f^{i l k} A_{\mu}^{l}(x)\left\langle j^{\mu, k}(x)\right\rangle=0
$$

Eq.(29-31) are identities for $j$. In above formula, we use the language of functional path integral and the expectation value is taken in a functional sense. We can translate path integral expressions into the language of quantum operator due to the exact correspondence between two approaches. Then the expectation operation is carried on the interacting vacuum state, i.e. that of the full Hamiltonian $H=H_{0}+H_{i}$ where $H_{i}$ denotes the interacting Hamiltonian. In above formula, all operators are in the Heisenberg picture. According to Gell-Mann-Low's theorem, we can rewrite them in the interacting picture: $\left\langle(\cdots)_{H}\right\rangle \equiv\left\langle\Omega\left|(\cdots)_{H}\right| \Omega\right\rangle=\left\langle 0\left|(\cdots)_{I} e^{-i \int d t H_{i I}(t)}\right| 0\right\rangle$ where $|\Omega\rangle$ is the vacuum state for the full Hamiltonian $H$ and $|0\rangle$ is that for the free $H_{0}$, and ' $H$ ' and 'I' denote the Heisenberg and interacting picture respectively.

Note that $\frac{\delta j}{\delta A}(x)$ and $j(x)$ are composite operators, so they should be understood as normal product in order to be well defined in taking their expectation values on the vacuum state. If we assume, $|\Omega\rangle \approx|0\rangle$, Eq.(29) is then: 


$$
D_{\mu}^{i j}[A](x)\left\langle 0\left|T\left[j^{\mu, j}(x) j^{\nu, k}(y)\right]\right| 0\right\rangle=0
$$

where we drop in $j$ linear terms of $Q$; Also we have used $\left\langle 0\left|\frac{\delta j^{\nu, k}}{\delta A_{\mu}^{j}}(x)\right| 0\right\rangle=0$ and $\left\langle 0\left|j^{\nu, l}(x)\right| 0\right\rangle=$ 0 because $\frac{\delta j^{\nu, k}}{\delta A_{\mu}^{j}}(x)$ and $j^{\nu, l}(x)$ are treated as normal products.

Because we notice that $\frac{\delta j}{\delta A}$ transforms in a good manner, similarly we can also add to the action a new source term, where $\frac{\delta j}{\delta A}$ couples to its external source, to obtain a new identity. For simplicity, we drop linear terms in $j$ which results in that $\frac{\delta j}{\delta A}$ is independent of $A$. Defining $\left(\frac{\delta j}{\delta A}\right)^{\mu \nu, a b} \stackrel{\text { def }}{=} \frac{\delta j^{\mu, a}}{\delta A_{\nu}^{b}}$, we can check the transformation rule: $\left(\frac{\delta j}{\delta A}\right)^{\prime \mu \nu, a b}=\left(U\left(\frac{\delta j}{\delta A}\right)^{\mu \nu} U^{-1}\right)^{a b}$. We add to the action a new source term $\tilde{Y}_{\mu \nu}^{b a}\left(\frac{\delta j}{\delta A}\right)^{\mu \nu, a b}$. To make the source term invariant under the gauge transformation, we assume that $\tilde{Y}$ transforms in the same way as $\left(\frac{\delta j}{\delta A}\right)$ does, i.e. $\tilde{Y}_{\mu \nu}^{\prime b a}=\left(U \tilde{Y}_{\mu \nu} U^{-1}\right)^{b a}$. The infinitesimal variation of $\tilde{Y}$ is then:

$$
\delta \tilde{Y}_{\mu \nu}^{b a}=-g \omega^{i}\left(f^{b i m} \tilde{Y}_{\mu \nu}^{m a}-f^{n i a} \tilde{Y}_{\mu \nu}^{b n}\right)
$$

The Ward identity becomes:

$$
\begin{aligned}
& D_{\mu}^{i j}[A] \frac{\delta W}{\delta A_{\mu}^{j}}+g\left(f^{b i m} \tilde{Y}_{\mu \nu}^{m a}-f^{n i a} \tilde{Y}_{\mu \nu}^{b n}\right) \frac{\delta W}{\delta \tilde{Y}_{\mu \nu}^{b a}} \\
& +g f^{i j k}\left(J_{\mu}^{j} \frac{\delta W}{\delta J_{\mu}^{k}}+\xi^{j} \frac{\delta W}{\delta \xi^{k}}+\bar{\xi}^{j} \frac{\delta W}{\delta \bar{\xi}^{k}}\right)=0
\end{aligned}
$$

Because $\frac{\delta j}{\delta A}$ is independent of $A$, only $\frac{\delta S}{\delta A}$ finally contributes to $\frac{\delta W}{\delta A}$. Repeating the same procedure as in the derivation of Eq.(29) and (30), we can obtain:

$$
\begin{aligned}
& D_{\mu}^{i j}[A](x)\left\langle T\left[j^{\mu, j}(x) \frac{\delta j^{\beta, l}}{\delta A_{\lambda}^{k}}(y)\right]\right\rangle \\
& +i g \delta^{4}(x-y)\left(f^{b i l}\left\langle\frac{\delta j^{\beta, k}}{\delta A_{\lambda}^{b}}(x)\right\rangle+f^{k i a}\left\langle\frac{\delta j^{\beta, a}}{\delta A_{\lambda}^{l}}(x)\right\rangle\right)=0 .
\end{aligned}
$$

The integration form is:

$$
g f^{i l j} \int d^{4} x A_{\mu}^{l}(x)\left\langle T\left[j^{\mu, j}(x) \frac{\delta j^{\beta, l}}{\delta A_{\lambda}^{k}}(y)\right]\right\rangle+i g\left(f^{b i l}\left\langle\frac{\delta j^{\beta, k}}{\delta A_{\lambda}^{b}}(x)\right\rangle+f^{k i a}\left\langle\frac{\delta j^{\beta, a}}{\delta A_{\lambda}^{l}}(y)\right\rangle\right)=0 .
$$

We can take derivative with respect to $Y$ for Eq.(28) arbitrary times and get a series of identities, each of them shows a relation among correlation functions for $j$ of the lower order and those of the higher order. This is similar to the BBKGY hierarchy in statistical physics [15].

\section{CURRENT AT LEADING ORDER}

In this section, as an example, we calculate the expectation value of the current $\left\langle j^{\mu, i}(x)\right\rangle$ at the leading order. Since $j^{\mu, i}(x)$ is a composite operator, in order to be well defined, it is treated as normal product. If the expectation value $j^{\mu, i}(x)$ is taken between the vacuum state of a free field Lagrange, we simply have $\left\langle 0\left|j^{\mu, i}(x)\right| 0\right\rangle=0$. So $\left\langle j^{\mu, i}(x)\right\rangle$ starts from the loop level. Here we consider the lowest order result at the one-loop level. At this level, 
the relevant term in the action is $-\int d^{4} x j_{\mu}^{i} A^{\mu, i}$. The corresponding term in $H_{i}$ is then $\int d^{3} x j_{\mu}^{i} A^{\mu, i}$, so $-i \int d t H_{i}=-i \int d^{4} x j_{\mu}^{i} A^{\mu, i}$. Hence we obtain:

$$
\left\langle j^{\mu, i}(x)\right\rangle=-i \int d^{4} y A_{\nu}^{j}(y)\left\langle 0\left|T\left[j_{A^{0}}^{\mu, i}(x) j_{A^{0}}^{\nu, j}(y)\right]\right| 0\right\rangle
$$

where $j_{A^{0}}$ is part of $j$ in Eq.(25), which is independent of $A$ and has only square terms of the $Q$ field; $-\int d^{4} y A_{\nu}^{j}(y) j_{A^{0}}^{\nu, j}(y)$ is part of the classical action which contributes to the lowest order. $j_{A^{0}}$ is given by:

$$
\begin{aligned}
j_{A^{0}}^{\mu, a}= & -g f^{a b c} \partial_{\nu}\left(Q^{\nu, b} Q^{\mu, c}\right)+g f^{a b c} Q^{\nu, c} \partial_{\nu} Q^{\mu, b}-g f^{a b c} Q^{\nu, c} \partial^{\mu} Q_{\nu}^{b} \\
& -\frac{1}{\alpha} g f^{a b c} Q^{\mu, c} \partial_{\nu} Q^{\nu, b}-g f^{a b c} \bar{C}^{b} \overleftarrow{⿱}^{\mu}{ }^{\mu} C^{c}+g f^{a b c} \bar{C}^{b} \partial^{\mu} C^{c}
\end{aligned}
$$

where we have not included terms proportional to $Q, Q Q Q$ and $Q \bar{C} C$. Because there are only square and cubic terms of $Q$ in $j$, linear terms of $Q$ in the action do not contribute to the result. We drop the $Q Q Q$ term both in $j$ and in the classical action because it contributes to the two-loop level.

Note that Eq.(37) should satisfy Eq.(31):

$$
\int d^{4} x f^{i l k} A_{\mu}^{l}(x)\left\langle j^{\mu, k}(x)\right\rangle=-i f^{i l k} \int d^{4} x d^{4} y A_{\mu}^{l}(x) A_{\nu}^{j}(y)\left\langle 0\left|T\left[j_{A^{0}}^{\mu, k}(x) j_{A^{0}}^{\nu, j}(y)\right]\right| 0\right\rangle=0
$$

Actually, for the leading-order $j$ given in Eq.(37), Eq.(30) and Eq.(31) are equivalent. Noticing in Eq.(31) the term $i f^{i l j} A_{\mu}^{l}(y)\left\langle\frac{\delta j^{\nu, k}}{\delta A_{\mu}^{j}}(y)\right\rangle$ gives an additional strong coupling constant $g$ relative to rest terms, we can neglect it for the leading-order $j$ and obtain:

$$
f^{i l j} \int d^{4} x A_{\mu}^{l}(x)\left\langle T\left[j_{A^{0}}^{\mu, j}(x) j_{A^{0}}^{\nu, k}(y)\right]\right\rangle+i f^{i k l}\left\langle j^{\nu, l}(y)\right\rangle=0
$$

where only $j_{A^{0}}$ contributes to the first term and other parts of $j$, i.e. $j_{A^{1}}$ and $j_{A^{2}}$, contribute to the higher order. Multiplying the above equation by $A_{\nu}^{k}(y)$ and integrating it over $y$, we obtain:

$$
f^{i l k} \int d^{4} x d^{4} y A_{\mu}^{l}(x) A_{\mu}^{m}(y)\left\langle T\left[j_{A^{0}}^{\mu, k}(x) j_{A^{0}}^{\nu, m}(y)\right]\right\rangle+i f^{i l k} \int d^{4} y A_{\nu}^{l}(y)\left\langle j^{\nu, k}(y)\right\rangle=0
$$

Due to Eq.(39), the above equation is actually:

$$
2 i f^{i l k} \int d^{4} y A_{\nu}^{l}(y)\left\langle j^{\nu, k}(y)\right\rangle=0
$$

Surely it is the same as Eq.(39) itself.

The self-energy tensor $\Pi^{\mu \nu, a b}(x, y)=\left\langle 0\left|T\left[j_{A^{0}}^{\mu, a}(x) j_{A^{0}}^{\nu, b}(y)\right]\right| 0\right\rangle$ corresponds to one-loop diagrams of the (quantum-)gluon and the ghost with two external $A$ legs(see fig.1). In momentum space, $\Pi^{\mu \nu, a b}(p)$ is given by:

$$
\begin{aligned}
& \Pi^{\mu \nu, a b}(p)=\Pi_{Q}^{\mu \nu, a b}(p)+\Pi_{C}^{\mu \nu, a b}(p), \\
& \Pi_{Q}^{\mu \nu, a b}(p)=i g^{2} C_{A} \delta^{a b}\left(g^{\mu \nu} p^{2}-p^{\mu} p^{\nu}\right) \frac{1}{(4 \pi)^{2}} \frac{10}{3}\left(\frac{1}{\epsilon_{r}}+\ln (4 \pi)-\gamma+\frac{59}{30}+\ln \left(\frac{\mu^{2}}{-p^{2}-i \epsilon}\right)\right), \\
& \Pi_{C}^{\mu \nu, a b}(p)=i g^{2} C_{A} \delta^{a b}\left(g^{\mu \nu} p^{2}-p^{\mu} p^{\nu}\right) \frac{1}{(4 \pi)^{2}} \frac{1}{3}\left(\frac{1}{\epsilon_{r}}+\ln (4 \pi)-\gamma+\frac{8}{3}+\ln \left(\frac{\mu^{2}}{-p^{2}-i \epsilon}\right)\right),
\end{aligned}
$$


where $\Pi_{Q}^{\mu \nu, a b}$ is from the gluon loop and $\Pi_{C}^{\mu \nu, a b}$ from the ghost loop; $C_{A}=N_{c}$ for $S U\left(N_{c}\right)$; $\mu^{2}$ is a mass scale; $\gamma$ is the Euler's constant; The pole term $1 / \epsilon_{r}=2-D / 2$ arises in the regularization. We calculate $\Pi_{Q}^{\mu \nu, a b}$ with the gauge parameter $\alpha=1$, i.e. we choose the Feynman gauge. The term $\frac{1}{\epsilon_{r}}+\ln (4 \pi)-\gamma$ is to be cancelled by the counterterm in $\overline{M S}$ scheme. After the reduction of the divergent part for $\Pi^{\mu \nu, a b}$, we obtain:

$$
\Pi^{\mu \nu, a b}(p)=i g^{2} \frac{1}{(4 \pi)^{2}} \frac{11}{3} C_{A} \delta^{a b}\left(g^{\mu \nu} p^{2}-p^{\mu} p^{\nu}\right) \ln \left(\frac{\mu^{2}}{-p^{2}-i \epsilon}\right) .
$$

Then we take the Fourier transformation and get $\Pi^{\mu \nu, a b}(x, y)$ :

$$
\begin{aligned}
\Pi^{\mu \nu, a b}(x, y) & =\int \frac{d^{4} p}{(2 \pi)^{4}} \Pi^{\mu \nu, a b}(p) \exp (-i p(x-y)) \\
& =-i g^{2} C_{A} \frac{1}{(4 \pi)^{2}} \frac{11}{3} \delta^{a b}\left(g^{\mu \nu} \partial^{2}-\partial^{\mu} \partial^{\nu}\right) H(x-y)
\end{aligned}
$$

where $\partial$ acts on $x$ or $y$ and $H(x)$ is the Fourier transformation of $\ln \left(\frac{\mu^{2}}{-p^{2}-i \epsilon}\right)$ and its derivation is given in Appendix B. Substituting Eq.(45) into Eq.(37), we derive:

$$
\left\langle j^{\mu, a}(x)\right\rangle=-g^{2} C_{A} \frac{1}{(4 \pi)^{2}} \frac{11}{3} \int d^{4} y H(x-y)\left(g^{\mu \nu} \partial^{2}-\partial^{\mu} \partial^{\nu}\right) A_{\nu}^{a}(y)
$$

where $\partial$ acts on $A(y)$.

It is interesting to compare Eq.(44) with the self-energy tensor of the quark loop with two external $A$ legs:

$$
\Pi_{q-\text { loop }}^{\mu \nu, a b}=-i g^{2} \frac{1}{(4 \pi)^{2}} \frac{2}{3} n_{f} \delta^{a b}\left(g^{\mu \nu} p^{2}-p^{\mu} p^{\nu}\right) \ln \left(\frac{\mu^{2}}{-p^{2}-i \epsilon}\right)
$$

Note that Eq.(44) and (47) have opposite signs. This is just the feature of the non-Abelian gauge field which is of the same origin as the asymptotic freedom and the confinement. It means that the current generated by the gluons is opposite in sign to that by the quark. In Eq.(44), we require $p^{2}<0$ to give a real current $j(x)$. If $p^{2}>0$, the current $j(x)$ has an imaginary part which would be related to the production rate for the real gluon pair.

In fact, in the derivation of $\Pi^{\mu \nu, a b}(p)$, there is a small positive number $i \epsilon$ in the gluon and ghost propagator. It finally appears in $\ln \frac{\mu^{2}}{-p^{2}-i \epsilon}$. Then we can get the real part of $\Pi^{\mu \nu, a b}(p)$ which corresponds to the production rate by noting $\operatorname{Im} \ln \frac{\mu^{2}}{-p^{2}-i \epsilon}=-\pi$. Similar to the definition of the production rate for $q \bar{q}$ in [14], we obtain the production rate for $g g$ :

$$
\begin{aligned}
R_{g g} & =\operatorname{Re} \int d^{4} p A_{\mu}^{a}(p) \Pi^{\mu \nu, a b}(p) A_{\nu}^{b}(-p) \\
& =\frac{1}{4} \frac{11}{3} C_{A} \alpha_{s} \int d^{4} p \theta\left(p^{2}\right)\left[p^{2}\left(A^{a}(p) \cdot A^{a}(-p)\right)-\left(p \cdot A^{a}(p)\right)\left(p \cdot A^{a}(-p)\right)\right]
\end{aligned}
$$

Comparing this rate with that for producing $q \bar{q}$ in the classical field:

$$
R_{q \bar{q}}=-\frac{1}{4} \frac{2}{3} n_{f} \alpha_{s} \int d^{4} p \theta\left(p^{2}\right)\left[p^{2}\left(A^{a}(p) \cdot A^{a}(-p)\right)-\left(p \cdot A^{a}(p)\right)\left(p \cdot A^{a}(-p)\right)\right]
$$

Note that $R_{q \bar{q}}$ has the same form and sign as $R_{e^{+} e^{-}}$, the rate for the electron-positron pair production in QED, while $R_{g g}$ has opposite sign. We remember that if $A_{c l} \rightarrow g g$ and $A_{c l} \rightarrow q \bar{q}$ are real physical processes, $R_{g g}$ and $R_{q \bar{q}}$ can be written as $R_{g g}=\left|M\left(A_{c l} \rightarrow g g\right)\right|^{2}$ 
and $R_{q \bar{q}}=\left|M\left(A_{c l} \rightarrow q \bar{q}\right)\right|^{2}$ respectively, therefore we have $R_{g g}, R_{q \bar{q}} \geq 0$, but from the above result Eq.(48,49) they have opposite signs. Hence, the leading order term in $g$ does not give the correct result for the $g g$ pair production. We note that the leading order (in $g$ ) gluon pair production term is not gauge invariant with respect to the gauge transformation given by Eq.(3). In order to obtain correct gauge invariant result for gluon pair production we have to add higher order terms in $g$, which contains three and four $A$ 's, into this result. For details about this topic, see [16].

Now we try to understand the physical implication of the current induced by the gluon. In the lowest order, Eq.(24) can be written as:

$$
D_{\nu}^{i j}[A(x)] F^{\nu \mu, j}[A(x)]=-g^{2} C_{A} \frac{1}{(4 \pi)^{2}} \frac{11}{3} \int d^{4} y H(x-y)\left(g^{\mu \nu} \partial^{2}-\partial^{\mu} \partial^{\nu}\right) A_{\nu}^{a}(y)
$$

In the lowest order, this equation of motion for $A$ corresponds to the effective action:

$$
\begin{aligned}
S_{e f f}[A]= & -\int d^{4} x \frac{1}{4} F_{\mu \nu}^{j}[A(x)] F^{\mu \nu, j}[A(x)] \\
& +g^{2} C_{A} \frac{1}{(4 \pi)^{2}} \frac{11}{6} \int d^{4} x d^{4} y A_{\mu}^{a}(x) H(x-y)\left(g^{\mu \nu} \partial^{2}-\partial^{\mu} \partial^{\nu}\right) A_{\nu}^{a}(y)
\end{aligned}
$$

We can write $S_{\text {eff }}[A]$ in momentum space as:

$$
\begin{aligned}
S_{e f f}[A]= & -\frac{1}{2} \int d^{4} p A_{\mu}^{a}(-p) A_{\nu}^{a}(p)\left(g^{\mu \nu} p^{2}-p^{\mu} p^{\nu}\right) \\
& \cdot\left[1+g^{2} C_{A} \frac{1}{(4 \pi)^{2}} \frac{11}{3} \ln \left(\frac{\mu^{2}}{-p^{2}-i \epsilon}\right)\right] \\
& + \text { square and cubic terms of } A(p)
\end{aligned}
$$

One can define a new amplitude $A^{\prime}(p)$ with the radiation correction caused by the gluon in the lowest order: $A^{\prime}(p)=[1+\kappa(p)]^{1 / 2} A(p)$ where $\kappa(p)=g^{2} \frac{1}{(4 \pi)^{2}} \frac{11}{3} C_{A} \ln \left(\frac{\mu^{2}}{-p^{2}-i \epsilon}\right)$. Therefore, the correction effect brought by the gluon fluctuation on the classical background field is similar to the medium screening effect. This screening effect becomes larger as $\left|p^{2}\right|$ decreases and lower as $\left|p^{2}\right|$ increases. This is just the feature of the effective coupling constant. In fact, since there is a relation between the renormalization constant $Z_{A}$ for $A$ and $Z_{g}$ for

$g: Z_{g}=Z_{A}^{-1 / 2}, g A$ should be unchanged after redefinition of the field and the coupling constant. Now we redefine the field as $A^{\prime}(p)=[1+\kappa(p)]^{1 / 2} A(p)$, accordingly we can redefine the coupling constant as $g_{\text {eff }}^{2}(p)=[1+\kappa(p)]^{-1} g^{2}(p)$. The above argument is made for the gluon situation. If we include the quark sector into the classical action, we should add a term to $\kappa(p): \kappa(p)=g^{2} \frac{1}{(4 \pi)^{2}}\left[\frac{11}{3} C_{A}-\frac{2}{3} n_{f}\right] \ln \left(\frac{\mu^{2}}{-p^{2}-i \epsilon}\right)$. It shows the screening effect brought by the quark is opposite to that by the gluon. We see that the effective coupling constant $g_{\text {eff }}^{2}(p)$ has a running property and shows the feature of asymptotic freedom. Expressed by the new field and the effective coupling constant $g_{\text {eff }}$, we can write the original field equation (50) as: $D_{\nu}^{i j}\left[A^{\prime}, g_{e f f}\right] F^{\nu \mu, j}\left[A^{\prime}, g_{e f f}\right]=0$. Therefore, the current induced by the quantum gluon is just the 'displacement' current in the polarized vacuum, and its effect is equivalent to redefinition of the field and the coupling constant.

\section{SUMMARY}

In summary, for a system which contain a classical chromofield and the gluon simultaneously, we derive the equation of motion for the classical chromofield and for the gluon. 
Furthermore, we prove that the equation for the classical field is the same as for the gluon in a functional path integral sense. The proof is not straightforward, it needs a symmetry property between the gauge fixing and ghost sectors of the full action. The inhomogeneous field equation contains a current term which is the expectation value of a composite operator including linear, square and cubic terms of the quantum field $Q$. We call the current as induced by the gluon. Note that this current essentially is classical quantity. We also derive the identity for this current. These identities represent the constraint on this current from the gauge invariance. We calculate the current at the leading order. In momentum space, it is just the contraction of the classical field $A(p)$ with the self energy tensor $\Pi(p)$ with two $A$ 's. The current generated by the gluon is opposite in sign to that generated by the quark. This is just the feature of the non-Abelian gauge field theory which is asymptotic free. We require $p^{2}<0$ to give a real current $j(x)$. If $p^{2}>0$, the current $j(x)$ has an imaginary part which would give the production rate for the real gluon pair. Physically, the current induced by the quantum gluon is just the 'displacement' current in the polarized vacuum, and its effect is equivalent to redefinition of the field and the coupling constant.

We emphasize that all the analysis done here is with respect to QCD vacuum where no medium effect is included yet. Normally one works in closed-time path integral formalism in order to derive a current in a medium. Then the current can be related to the nonequilibrium distribution function of the gluon. We plan to address this issue in the future where we wish to combine closed-time path integral formalism with background field method of QCD.

\section{ACKNOWLEDGMENTS}

Q.W., C.W.K. and G.C.N. acknowledge the financial support from Alexander von Humboldt Foundation. Q.W. is also supported in part by the National Natural Science Foundation of China. 


\section{FIGURES}

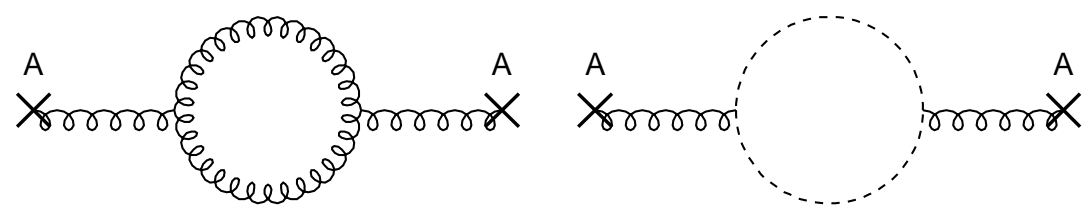

FIG. 1. The one-loop diagrams for the self-energy with two external $A$ legs. One diagram is the gluon loop(screw line) and the other for the ghost loop(dashed line).

\section{Appendix A.}

In case II, since all sources are functionals of two independent variables $A$ and $Y$, the Ward identity (26) changes to:

$$
D_{\mu}^{i j}[A] \frac{\delta W}{\delta A_{\mu}^{j}}+g f^{i j k} Y_{\mu}^{j} \frac{\delta W}{\delta Y_{\mu}^{k}}=0
$$

where $\delta W / \delta A_{\mu}^{j}$ and $\delta W / \delta Y_{\mu}^{k}$ are given by:

$$
\begin{aligned}
\frac{\delta W}{\delta A_{\mu}^{j}}= & D_{\nu}^{j k}[A] F^{\nu \mu, k}[A]-\left\langle j^{\mu, j}\right\rangle+Y_{\nu}^{k}\left\langle\frac{\delta j^{\nu, k}}{\delta A_{\mu}^{j}}\right\rangle \\
& +\frac{\delta J_{\nu}^{k}}{\delta A_{\mu}^{j}}\left\langle Q^{\nu, k}\right\rangle+\frac{\delta \bar{\xi}^{k}}{\delta A_{\mu}^{j}}\left\langle C^{k}\right\rangle+\left\langle\bar{C}^{k}\right\rangle \frac{\delta \xi^{k}}{\delta A_{\mu}^{j}} \\
\frac{\delta W}{\delta Y_{\mu}^{k}}= & \left\langle j^{\mu, k}\right\rangle+\frac{\delta J_{\nu}^{m}}{\delta Y_{\mu}^{k}}\left\langle Q^{\nu, m}\right\rangle+\frac{\delta \bar{\xi}^{m}}{\delta Y_{\mu}^{k}}\left\langle C^{m}\right\rangle+\left\langle\bar{C}^{m}\right\rangle \frac{\delta \xi^{m}}{\delta Y_{\mu}^{k}}
\end{aligned}
$$

Since all the field averages are set to zero during our derivation, $\frac{\delta W}{\delta A_{\mu}^{j}}$ is the same as Eq.(27) and $\frac{\delta W}{\delta Y_{\mu}^{k}}=\left\langle j^{\mu, k}\right\rangle$. So we also get Eq.(30).

\section{Appendix B.}

To derive the Fourier transform of $\ln \left[-p^{2}-i \epsilon\right]$, we set:

$$
I(x, \xi) \equiv \int \frac{d^{4} p}{(2 \pi)^{4}} e^{-i p \cdot x} \ln \left[-p_{0}^{2}+\xi^{2}|\vec{p}|^{2}-i \epsilon\right] .
$$

To calculate $I(x, \xi)$, we first evaluate the following function $J(x, \xi)$ :

$$
2 \xi J(x, \xi) \equiv \frac{d I(x, \xi)}{d \xi}=\int \frac{d^{4} p}{(2 \pi)^{4}} e^{-i p \cdot x} \frac{2 \xi|\vec{p}|^{2}}{-p_{0}^{2}+\xi^{2}|\vec{p}|^{2}-i \epsilon}
$$

First integrate over $p_{0}$ :

$$
\begin{aligned}
J(x, \xi) & =-\int \frac{d^{3} p}{(2 \pi)^{3}} \int \frac{d p_{0}}{(2 \pi)} \frac{e^{-i p_{0} x^{0}+i \vec{p} \cdot \vec{x}}|\vec{p}|^{2}}{\left(p_{0}-\xi|\vec{p}|+i \epsilon\right)\left(p_{0}+\xi|\vec{p}|-i \epsilon\right)} \\
& =i \theta\left(x^{0}\right) \int \frac{d^{3} p}{(2 \pi)^{3}} \frac{e^{-i \xi|\vec{p}| x^{0}+i \vec{p} \cdot \vec{x}}}{2 \xi|\vec{p}|}|\vec{p}|^{2}+i \theta\left(-x^{0}\right) \int \frac{d^{3} p}{(2 \pi)^{3}} \frac{e^{i \xi|\vec{p}| x^{0}+i \vec{p} \cdot \vec{x}}}{2 \xi|\vec{p}|}|\vec{p}|^{2}
\end{aligned}
$$


Then integrate angular variables:

$$
\begin{aligned}
2 \xi J(x, \xi)= & \theta\left(x^{0}\right) \int_{0}^{\infty} \frac{p^{2} d p}{(2 \pi)^{2}} \frac{e^{-i \xi p x^{0}}}{|\vec{x}|}\left(e^{i p|\vec{x}|}-e^{-i p|\vec{x}|}\right) \\
& +\theta\left(-x^{0}\right) \int_{0}^{\infty} \frac{p^{2} d p}{(2 \pi)^{2}} \frac{e^{i \xi p x^{0}}}{|\vec{x}|}\left(e^{i p|\vec{x}|}-e^{-i p|\vec{x}|}\right)
\end{aligned}
$$

Next, integrate with $\xi$ :

$$
\begin{aligned}
I(x, \xi)= & \frac{\theta\left(x^{0}\right)}{|\vec{x}|} \int_{0}^{\infty} \frac{p^{2} d p}{(2 \pi)^{2}} \frac{e^{-i \xi p x^{0}}}{-i p x^{0}}\left(e^{i p|\vec{x}|}-e^{-i p|\vec{x}|}\right) \\
& +\frac{\theta\left(-x^{0}\right)}{|\vec{x}|} \int_{0}^{\infty} \frac{p^{2} d p}{(2 \pi)^{2}} \frac{e^{i \xi p x^{0}}}{i p x^{0}}\left(e^{i p|\vec{x}|}-e^{-i p|\vec{x}|}\right) \\
= & -\frac{\theta\left(x^{0}\right)}{|\vec{x}|\left(x^{0}\right)^{2}} \frac{d}{d \xi} \int_{0}^{\infty} \frac{d p}{(2 \pi)^{2}} e^{-i \xi p x^{0}}\left(e^{i p|\vec{x}|}-e^{-i p|\vec{x}|}\right) \\
& -\frac{\theta\left(-x^{0}\right)}{|\vec{x}|\left(x^{0}\right)^{2}} \frac{d}{d \xi} \int_{0}^{\infty} \frac{d p}{(2 \pi)^{2}} e^{i \xi p x^{0}}\left(e^{i p|\vec{x}|}-e^{-i p|\vec{x}|}\right) \\
= & \frac{-i}{2 \pi^{2}}\left[\frac{\theta\left(x^{0}\right)}{\left.|\vec{x}|^{\left(x^{0}\right.}\right)^{2}} \cdot \frac{4 \xi\left(x^{0}\right)^{2}|\vec{x}|}{\left[|\vec{x}|^{2}-\xi^{2}\left(x^{0}\right)^{2}+i \epsilon\right]^{2}}\right. \\
& \left.+\frac{\theta\left(-x^{0}\right)}{|\vec{x}|\left(x^{0}\right)^{2}} \cdot \frac{4 \xi\left(x^{0}\right)^{2}|\vec{x}|}{\left[|\vec{x}|^{2}-\xi^{2}\left(x^{0}\right)^{2}-i \epsilon\right]^{2}}\right]
\end{aligned}
$$

The final result becomes:

$$
I(x, \xi)=\frac{-i \xi}{\pi^{2}}\left[\frac{\theta\left(x_{0}\right)}{\left[|\vec{x}|^{2}-\xi^{2}\left(x^{0}\right)^{2}+i \epsilon\right]^{2}}+\frac{\theta\left(-x_{0}\right)}{\left[|\vec{x}|^{2}-\xi^{2}\left(x^{0}\right)^{2}-i \epsilon\right]^{2}}\right]
$$

Taking $\xi=1$, we get:

$$
\begin{aligned}
& \int \frac{d^{4} p}{(2 \pi)^{4}} e^{-i p \cdot x} \ln \left[-p_{0}^{2}+|\vec{p}|^{2}-i \epsilon\right] \\
& =\frac{-i}{\pi^{2}}\left[\frac{\theta\left(x_{0}\right)}{\left[|\vec{x}|^{2}-\left(x^{0}\right)^{2}+i \epsilon\right]^{2}}+\frac{\theta\left(-x_{0}\right)}{\left[|\vec{x}|^{2}-\left(x^{0}\right)^{2}-i \epsilon\right]^{2}}\right]
\end{aligned}
$$

Then $H(x)$ is given by:

$$
\begin{aligned}
H(x)= & \int \frac{d^{4} p}{(2 \pi)^{4}} e^{-i p \cdot x} \ln \left[\frac{\mu^{2}}{-p^{2}-i \epsilon}\right]=\delta(x) \ln \mu^{2} \\
& -\frac{i}{\pi^{2}}\left[\frac{\theta\left(x_{0}\right)}{\left[|\vec{x}|^{2}-\left(x^{0}\right)^{2}+i \epsilon\right]^{2}}+\frac{\theta\left(-x_{0}\right)}{\left[|\vec{x}|^{2}-\left(x^{0}\right)^{2}-i \epsilon\right]^{2}}\right]
\end{aligned}
$$




\section{REFERENCES}

[1] see, e.g., G. Z. Zhou, Z. B. Su, B. L. Hao and L. Yu, Phys. Rep. 118, 1(1985); E. Calzetta and B. L. Hu, Phys. Rev. D37, 2878(1988).

[2] H-T Elze and U. Heinz, Phys. Rep. 183, 81(1989).

[3] S.K. Wong, Nuovo Cimento A 65, 689(1970).

[4] Proceedings of 14th International Conference on Ultra-relativistic Nucleus-Nucleus Collisions (Quark Matter 99), Torino, Italy, 10-15 May 1999 (To appear in Nucl. Phys. A).

[5] K. J. Eskola and M. Gyulassy, Phys. Rev. C47 (1993) 2329; R. S. Bhalerao and G. C. Nayak, Phys. Rev. C 61 (2000) 054907.

[6] L. McLerran and R. Venugopalan, Phys. Rev. D49, 2233(1994); D49, 3352(1994); D50, 2225(1994);

[7] B. S. DeWitt, Phys. Rev. 162, 1195 and 1239(1967); in Dynamic theory of groups and fields (Gordon and Breach, 1965).

[8] G. 't Hooft, Nucl. Phys. B62, 444(1973).

[9] L. F. Abbott, Nucl. Phys. B185, 189(1981).

[10] R. B. Sohn, Nucl. Phys. B273, 468(1986).

[11] H. Kluberg-Stern and J. B. Zuber, Phys. Rev. D12, 482(1975).

[12] B. W. Lee and J. Zinn-Justin, Phys. Rev. D7, 1049(1973).

[13] H.-Th. Elze, Z. Phys. C47, 647(1990).

[14] C. Itzykson and J.-B. Zuber, Quantum Field Theory(McGraw-Hill, 1985), p.185,323.

[15] R. Balescu, Equilibrium and Non-equilibrium Statistical Physics(Wiley, New York, 1973).

[16] D. D. Dietrich, G. C. Nayak and W. Greiner, hep-ph/0009178; hep-th/0007139; Submitted to Phys. Rev. D. 